\title{
Some Nonlinear Delay Volterra-Fredholm Type Dynamic Integral Inequalities on Time Scales
}

\author{
Yazhou Tian $\mathbb{D},{ }^{1}$ A. A. El-Deeb, ${ }^{2}$ and Fanwei Meng $\mathbb{D}^{3}$ \\ ${ }^{1}$ School of Mathematical Sciences, University of Jinan, Jinan, Shandong 250022, China \\ ${ }^{2}$ Department of Mathematics, Faculty of Science, Al-Azhar University, Nasr City, Cairo 11884, Egypt \\ ${ }^{3}$ School of Mathematical Sciences, Qufu Normal University, Qufu, Shandong 273165, China
}

Correspondence should be addressed to Yazhou Tian; tianyazhou369@163.com

Received 22 February 2018; Accepted 25 July 2018; Published 12 August 2018

Academic Editor: Manuel De la Sen

Copyright (C) 2018 Yazhou Tian et al. This is an open access article distributed under the Creative Commons Attribution License, which permits unrestricted use, distribution, and reproduction in any medium, provided the original work is properly cited.

We are devoted to studying a class of nonlinear delay Volterra-Fredholm type dynamic integral inequalities on time scales, which can provide explicit bounds on unknown functions. The obtained results can be utilized to investigate the qualitative theory of nonlinear delay Volterra-Fredholm type dynamic equations. An example is also presented to illustrate the theoretical results.

\section{Introduction}

Since Hilger established the theory of time scales [1], it has become the research focus of mathematics and engineering field [2]. Particularly, integral inequalities play an important role in studying the qualitative properties of dynamic equations on time scales. For example, the integral inequalities were employed to investigate the stability of switched systems or uncertain nonlinear systems $[3,4]$.

In recent years, many authors have been devoted to studying different kinds of integral inequalities and their applications [5-24], especially the application of Volterra-Fredholm integrodifferential system [25-28]. To mention a few, in [8], $\mathrm{Gu}$ and Meng considered the nonlinear dynamic integral inequalities on time scales and applied the theoretical results to Volterra-Fredholm integrodifferential system, and Liu [9] investigated the linear delay Volterra-Fredholm type dynamic integral inequalities which generalized the main results of [8]. In [20], $\mathrm{Xu}$ and $\mathrm{Ma}$ considered Volterra-Fredholm type integral inequalities in two independent variables and their applications in partial differential equations. Very recently, in [22], Ding and Ahmad studied Volterra-Fredholm type integral inequalities and their applications to fractional differential equations. As is known to us, few authors pay attention to nonlinear delay Volterra-Fredholm type dynamic integral inequalities on time scales. This is the main reason why we establish this topic.

This paper investigates a class of nonlinear dynamic integral inequalities on time scales, which can be utilized as effective tools in the study of delay Volterra-Fredholm type dynamic equations. At the end, we provide an example to illustrate the main results.

\section{Main Results}

Throughout the paper, let $\mathbb{R}$ be the set of real numbers, $\mathbb{R}_{+}=[0, \infty), C(M, S)$ be the class of all continuous functions defined on set $M$ with range in the set $S$, and $\mathbb{T}$ be an arbitrary time scale. The set $\mathbb{T}^{k}$ is derived from $\mathbb{T}$ as follows: if $\mathbb{T}$ has a left-scattered maximum $n$, then $\mathbb{T}^{k}=\mathbb{T}-\{n\}$; otherwise, $\mathbb{T}^{k}=\mathbb{T} . I=\left[t_{0}, T_{1}\right] \cap \mathbb{T}^{k}$, where $t_{0} \in \mathbb{T}^{k}, T_{1} \in \mathbb{T}^{k}$, and $T_{1}>t_{0}$. $C_{r d}$ denotes the set of rd-continuous functions. $\mathfrak{R}$ represents the set of all regressive and rd-continuous functions, and $\Re^{+}=\{p \in \mathfrak{R}: 1+\mu(t) p(t)>0, t \in \mathbb{T}\}$. The graininess function $\mu: \mathbb{T} \longrightarrow[0, \infty)$ is defined by $\mu(t):=\sigma(t)-t$, the forward jump operator $\sigma: \mathbb{T} \longrightarrow \mathbb{T}$ by $\sigma(t)=\inf \{s \in$ $\mathbb{T}: s>t\}$, and the circle plus addition $\oplus$ is defined by $(p \oplus q)(t)=p(t)+q(t)+\mu(t) p(t) q(t), t \in \mathbb{T}^{k}$.

Next, we introduce some lemmas to establish the main results. 
Lemma 1 (comparison theorem [2]). Let $u, b \in C_{r d}$ and $a \in$ $\mathfrak{R}^{+}$. If

$$
u^{\Delta}(t) \leq a(t) u(t)+b(t), \quad t \geq t_{0}, t \in \mathbb{T}^{k},
$$

then

$$
u(t) \leq u\left(t_{0}\right) e_{a}\left(t, t_{0}\right)+\int_{t_{0}}^{t} b(\tau) e_{a}(t, \sigma(\tau)) \Delta \tau,
$$

$$
t \geq t_{0}, t \in \mathbb{T}^{k} \text {. }
$$

Lemma 2 (see [2]). Assume that $w: \mathbb{T} \times \mathbb{T}^{k} \longrightarrow \mathbb{R}_{+}$is continuous at $(t, t)$ with $t>t_{0}$ and $t_{0}, t \in \mathbb{T}^{k}$, and $w(t, \cdot)$ is $r d$-continuous on $\left[t_{0}, \sigma(t)\right]$. For any $\varepsilon>0$, if there exists a neighborhood $\mathfrak{U}$ of $t$, independent of $\xi \in\left[t_{0}, \sigma(t)\right]$, such that

$$
\begin{aligned}
& \left|w(\sigma(t), \xi)-w(s, \xi)-w_{t}^{\Delta}(t, \xi)(\sigma(t)-s)\right| \\
& \quad \leq \varepsilon|\sigma(t)-s|, \quad s \in \mathcal{U},
\end{aligned}
$$

where $w_{t}^{\Delta}$ represents the derivative of $\omega$ with respect to $t$, then

$$
v(t)=\int_{t_{0}}^{t} w(t, \xi) \Delta \xi
$$

implies

$$
v^{\Delta}(t)=\int_{t_{0}}^{t} w_{t}^{\Delta}(t, \xi) \Delta \xi+w(\sigma(t), t) .
$$

Lemma 3 (see [6]). Let $a \geq 0$ and $p \geq q>0$. For any $K>0$, we have

$$
a^{q / p} \leq \frac{q}{p} K^{(q-p) / p} a+\frac{p-q}{p} K^{q / p} .
$$

Theorem 4. Assume that $p \geq q>0, p \geq r>0$, and $\lambda \geq$ 0 are positive constants, $u, a, b, f_{1}, f_{2}, g: I \longrightarrow \mathbb{R}_{+}$are $r d$ continuous functions, $a$ is nondecreasing, $\tau: I \longrightarrow I, \tau(t) \leq t$, $-\infty<\alpha=\inf \{\tau(t), t \in I\} \leq t_{0}$, and $\varphi \in C_{r d}\left(\left[\alpha, t_{0}\right] \cap \mathbb{T}, \mathbb{R}_{+}\right)$. If $u$ satisfies

$$
\begin{aligned}
& u^{p}(t) \leq a(t)+b(t) \int_{t_{0}}^{t}\left[f_{1}(s) u^{q}(\tau(s))\right. \\
& \left.+f_{2}(s) \int_{t_{0}}^{s} g(\xi) u^{r}(\xi) \Delta \xi\right] \Delta s+\lambda b\left(T_{1}\right) \\
& \cdot \int_{t_{0}}^{T_{1}}\left[f_{1}(s) u^{q}(\tau(s))\right. \\
& \left.+f_{2}(s) \int_{t_{0}}^{s} g(\xi) u^{r}(\xi) \Delta \xi\right] \Delta s, \quad t \in I
\end{aligned}
$$

with the initial condition

$$
\begin{aligned}
u(t) & =\varphi(t), \quad \text { for } t \in\left[\alpha, t_{0}\right], \\
\varphi(\tau(t)) & \leq a^{1 / p}(t) \quad \text { for every } t \in I \text { with } \tau(t) \leq t_{0},
\end{aligned}
$$

then

$$
\begin{gathered}
u(t) \leq\left\{D\left(\lambda, T_{1}\right) e_{A \oplus B}\left(t, t_{0}\right)\right. \\
\left.+\int_{t_{0}}^{t} e_{A \oplus B}(t, \sigma(s)) C(s) \Delta s\right\}^{1 / p}
\end{gathered}
$$

for any $K>0, t \in I$

under the condition that $e_{A \oplus B}\left(T_{1}, t_{0}\right)<(\lambda+1) / \lambda$ and $\mu(t) B_{1}(t)<1$, where

$$
\begin{aligned}
& A(t)=b(t)\left[\frac{q}{p} K^{(q-p) / p} f_{1}(t)+\frac{r}{p} K^{(r-p) / p} f_{2}(t)\right. \\
& \left.\cdot \int_{t_{0}}^{t} g(\xi) \Delta \xi\right], \\
& B(t)=\frac{B_{1}(t)}{1-\mu(t) B_{1}(t)}, \\
& C(t)=[1+\mu(t) B(t)] C_{1}(t), \\
& B_{1}(t)=b^{\Delta}(t) \int_{t_{0}}^{\sigma(t)}\left[\frac{q}{p} K^{(q-p) / p} f_{1}(s)\right. \\
& \left.\quad+\frac{r}{p} K^{(r-p) / p} f_{2}(s) \int_{t_{0}}^{s} g(\xi) \Delta \xi\right] \Delta s, \\
& C_{1}(t)=b^{\Delta}(t) \int_{t_{0}}^{\sigma(t)}\left[\frac{p-q}{p} K^{q / p} f_{1}(s)\right. \\
& \left.\quad+\frac{p-r}{p} K^{r / p} f_{2}(s) \int_{t_{0}}^{s} g(\xi) \Delta \xi\right] \Delta s+b(t)\left[\frac{p-q}{p}\right. \\
& \left.\quad \cdot K^{q / p} f_{1}(t)+\frac{p-r}{p} K^{r / p} f_{2}(t) \int_{t_{0}}^{t} g(\xi) \Delta \xi\right], \\
& D\left(\lambda, T_{1}\right)=\frac{a\left(T_{1}\right)+\lambda \int_{t_{0}}^{T_{1}} e_{A \oplus B}\left(T_{1}, \sigma(s)\right) C(s) \Delta s}{\lambda+1-\lambda e_{A \oplus B}\left(T_{1}, t_{0}\right)} .
\end{aligned}
$$

Proof. Define $y(t)$ by

$$
\begin{aligned}
& y(t)=a\left(T_{1}\right)+b(t) \\
& \cdot \int_{t_{0}}^{t}\left[f_{1}(s) u^{q}(\tau(s))+f_{2}(s) \int_{t_{0}}^{s} g(\xi) u^{r}(\xi) \Delta \xi\right] \Delta s \\
& +\lambda b\left(T_{1}\right) \\
& \cdot \int_{t_{0}}^{T_{1}}\left[f_{1}(s) u^{q}(\tau(s))+f_{2}(s) \int_{t_{0}}^{s} g(\xi) u^{r}(\xi) \Delta \xi\right] \\
& \cdot \Delta s, \quad t \in I .
\end{aligned}
$$

Then, $y(t) \geq 0, y(t)$ is nondecreasing,

$$
u(t) \leq y^{1 / p}(t), \quad t \in I,
$$


and

$$
\begin{aligned}
y\left(t_{0}\right) & =a\left(T_{1}\right)+\lambda b\left(T_{1}\right) \\
\cdot \int_{t_{0}}^{T_{1}} & {\left[f_{1}(s) u^{q}(\tau(s))+f_{2}(s) \int_{t_{0}}^{s} g(\xi) u^{r}(\xi) \Delta \xi\right] }
\end{aligned}
$$

$\cdot \Delta s$.

Next, we will prove that, for every $t \in I, u(\tau(t)) \leq y^{1 / p}(t)$.

Case 1. When $\tau(t)>t_{0}, t \in I$, we obtain

$$
u(\tau(t)) \leq y^{1 / p}(\tau(t)) \leq y^{1 / p}(t) .
$$

Case 2. For $t \in I$ with $\tau(t) \leq t_{0}$, by the initial condition (8), we have

$$
u(\tau(t))=\varphi(\tau(t)) \leq a^{1 / p}(t) \leq a^{1 / p}\left(T_{1}\right) \leq y^{1 / p}(t) .
$$

From (14) and (15), we always have the relation

$$
u(\tau(t)) \leq y^{1 / p}(t)
$$

By simple computation, it follows from (12) and (16) that

$$
\begin{aligned}
& y^{\Delta}(t)=b^{\Delta}(t) \int_{t_{0}}^{\sigma(t)}\left[f_{1}(s) u^{q}(\tau(s))\right. \\
& \left.+f_{2}(s) \int_{t_{0}}^{s} g(\xi) u^{r}(\xi) \Delta \xi\right] \Delta s+b(t)\left[f_{1}(t)\right. \\
& \left.\cdot u^{q}(\tau(t))+f_{2}(t) \int_{t_{0}}^{t} g(\xi) u^{r}(\xi) \Delta \xi\right] \leq b^{\Delta}(t) \\
& \cdot \int_{t_{0}}^{\sigma(t)}\left[f_{1}(s) y^{q / p}(s)\right. \\
& \left.+f_{2}(s) \int_{t_{0}}^{s} g(\xi) y^{r / p}(\xi) \Delta \xi\right] \Delta s+b(t)\left[f_{1}(t)\right. \\
& \left.\cdot y^{q / p}(t)+f_{2}(t) \int_{t_{0}}^{t} g(\xi) y^{r / p}(\xi) \Delta \xi\right] .
\end{aligned}
$$

For any $K>0$, it follows from Lemma 3 that

$$
\begin{aligned}
y^{q / p}(t) & \leq \frac{q}{p} K^{(q-p) / p} y(t)+\frac{p-q}{p} K^{q / p}, \\
y^{r / p}(t) & \leq \frac{r}{p} K^{(r-p) / p} y(t)+\frac{p-r}{p} K^{r / p} .
\end{aligned}
$$

This together with (17) implies

$$
\begin{aligned}
& y^{\Delta}(t) \leq b^{\Delta}(t) \int_{t_{0}}^{\sigma(t)}\left[f_{1}(s) y^{q / p}(s)\right. \\
& \left.+f_{2}(s) \int_{t_{0}}^{s} g(\xi) y^{r / p}(\xi) \Delta \xi\right] \Delta s+b(t)\left[f_{1}(t)\right. \\
& \left.\cdot y^{q / p}(t)+f_{2}(t) \int_{t_{0}}^{t} g(\xi) y^{r / p}(\xi) \Delta \xi\right] \leq y(\sigma(t)) \\
& \cdot b^{\Delta}(t) \int_{t_{0}}^{\sigma(t)}\left[\frac{q}{p} K^{(q-p) / p} f_{1}(s)\right. \\
& \left.+\frac{r}{p} K^{(r-p) / p} f_{2}(s) \int_{t_{0}}^{s} g(\xi) \Delta \xi\right] \Delta s+y(t) b(t)\left[\frac{q}{p}\right. \\
& \left.\cdot K^{(q-p) / p} f_{1}(t)+\frac{r}{p} K^{(r-p) / p} f_{2}(t) \int_{t_{0}}^{t} g(\xi) \Delta \xi\right] \\
& +b^{\Delta}(t) \int_{t_{0}}^{\sigma(t)}\left[\frac{p-q}{p} K^{q / p} f_{1}(s)\right. \\
& \left.+\frac{p-r}{p} K^{r / p} f_{2}(s) \int_{t_{0}}^{s} g(\xi) \Delta \xi\right] \Delta s+b(t)\left[\frac{p-q}{p}\right. \\
& \left.\cdot K^{q / p} f_{1}(t)+\frac{p-r}{p} K^{r / p} f_{2}(t) \int_{t_{0}}^{t} g(\xi) \Delta \xi\right] \\
& =A(t) y(t)+B_{1}(t) y(\sigma(t))+C_{1}(t)=A(t) y(t) \\
& +\frac{B(t)}{1+\mu(t) B(t)}\left(y(t)+\mu(t) y^{\Delta}(t)\right)+C_{1}(t),
\end{aligned}
$$

which yields

$$
\begin{aligned}
\frac{1}{1+\mu(t) B(t)} y^{\Delta}(t) \leq & \left(A(t)+\frac{B(t)}{1+\mu(t) B(t)}\right) y(t) \\
& +C_{1}(t),
\end{aligned}
$$

i.e.,

$$
y^{\Delta}(t) \leq(A \oplus B)(t) y(t)+C(t), \quad t \in I ;
$$

where $C(t)=[1+\mu(t) B(t)] C_{1}(t)$. Note that $y, C \in C_{r d}$ and $A \oplus B \in \Re^{+}$. By Lemma 1 , we get

$$
y(t) \leq y\left(t_{0}\right) e_{A \oplus B}\left(t, t_{0}\right)+\int_{t_{0}}^{t} e_{A \oplus B}(t, \sigma(s)) C(s) \Delta s,
$$

$t \in I$.

From (11) and (13), we get

$$
\begin{aligned}
& \frac{\lambda+1}{\lambda} y\left(t_{0}\right)-\frac{1}{\lambda} a\left(T_{1}\right)=y\left(T_{1}\right) \\
& \leq y\left(t_{0}\right) e_{A \oplus B}\left(T_{1}, t_{0}\right) \\
& \quad+\int_{t_{0}}^{T_{1}} e_{A \oplus B}\left(T_{1}, \sigma(s)\right) C(s) \Delta s ;
\end{aligned}
$$


i.e.,

$$
y\left(t_{0}\right) \leq D\left(\lambda, T_{1}\right) .
$$

Therefore,

$$
\begin{aligned}
y(t) \leq & D\left(\lambda, T_{1}\right) e_{A \oplus B}\left(t, t_{0}\right) \\
& +\int_{t_{0}}^{t} e_{A \oplus B}(t, \sigma(s)) C(s) \Delta s, \quad t \in I .
\end{aligned}
$$

Noting that $u(t) \leq y^{1 / p}(t)$, we obtain the required inequality (9). The proof is complete.

Remark 5. [8, Theorem 3] is the special case of Theorem 4 with $a(t) \equiv u_{0}, b(t) \equiv 1, \lambda=p=q=r=1$, and $\tau(t)=t$.

Remark 6. Theorem 4 generalizes [9, Theorem 3.1] to the nonlinear case.

Remark 7. From (9), we can obtain that the upper bound of $u(t)$ depends on parameters $p, q, r$, and $\lambda$. For practical system, the above parameters are easily to be obtained; therefore the theoretical upper bound can be computed by (9).

If we take $\lambda=1 / b\left(T_{1}\right), b\left(T_{1}\right) \neq 0$, then the following result can be obtained.

Corollary 8. Let $p, q, r, u, a, b, f_{1}, f_{2}, g, \tau, \varphi$ be defined as in Theorem 4 and $u$ satisfy

$$
\begin{aligned}
& u^{p}(t) \leq a(t)+b(t) \\
& \cdot \int_{t_{0}}^{t}\left[f_{1}(s) u^{q}(\tau(s))+f_{2}(s) \int_{t_{0}}^{s} g(\xi) u^{r}(\xi) \Delta \xi\right] \\
& \cdot \Delta s \\
& +\int_{t_{0}}^{T_{1}}\left[f_{1}(s) u^{q}(\tau(s))+f_{2}(s) \int_{t_{0}}^{s} g(\xi) u^{r}(\xi) \Delta \xi\right] \\
& \cdot \Delta s, \quad t \in I
\end{aligned}
$$

and (8). If $e_{A \oplus B}\left(T_{1}, t_{0}\right)<1+b\left(T_{1}\right)$ and $\mu(t) B_{1}(t)<1$, then

$$
\begin{gathered}
u(t) \leq\left\{\bar{D}\left(\lambda, T_{1}\right) e_{A \oplus B}\left(t, t_{0}\right)\right. \\
\left.+\int_{t_{0}}^{t} e_{A \oplus B}(t, \sigma(s)) C(s) \Delta s\right\}^{1 / p}
\end{gathered}
$$

for any $K>0, t \in I$,

where $A(t), B(t), C(t), B_{1}(t), C_{1}(t)$ are defined as in Theorem 4 and

$$
\begin{aligned}
\bar{D}\left(\lambda, T_{1}\right) \\
=\frac{a\left(T_{1}\right) b\left(T_{1}\right)+\int_{t_{0}}^{T_{1}} e_{A \oplus B}\left(T_{1}, \sigma(s)\right) C(s) \Delta s}{1+b\left(T_{1}\right)-e_{A \oplus B}\left(T_{1}, t_{0}\right)} .
\end{aligned}
$$

Theorem 9. Suppose that $p \geq q>0, p \geq r>0$, and $\lambda \geq 0$ are positive constants, $u, a, b, g: I \longrightarrow \mathbb{R}_{+}$are $r d$-continuous functions, $a$ is nondecreasing, $v(t, s), w(t, s)$ are defined as in Lemma 2 such that $v_{t}^{\Delta}(t, s) \geq 0$ and $w_{t}^{\Delta}(t, s) \geq 0$ for $t \geq s$, $\tau: I \longrightarrow I, \tau(t) \leq t,-\infty<\alpha=\inf \{\tau(t), t \in I\} \leq t_{0}$, and $\varphi \in C_{r d}\left(\left[\alpha, t_{0}\right] \cap \mathbb{T}, \mathbb{R}_{+}\right)$. If u satisfies

$$
\begin{aligned}
& u^{p}(t) \leq a(t)+b(t) \int_{t_{0}}^{t}\left[v(t, s) u^{q}(\tau(s))\right. \\
& \left.+w(t, s) \int_{t_{0}}^{s} g(\xi) u^{r}(\xi) \Delta \xi\right] \Delta s+\lambda b\left(T_{1}\right) \\
& \quad \cdot \int_{t_{0}}^{T_{1}}\left[v\left(T_{1}, s\right) u^{q}(\tau(s))\right. \\
& \left.+w\left(T_{1}, s\right) \int_{t_{0}}^{s} g(\xi) u^{r}(\xi) \Delta \xi\right] \Delta s, \quad t \in I
\end{aligned}
$$

and (8), then

$$
\begin{gathered}
u(t) \leq\left\{\widetilde{D}\left(\lambda, T_{1}\right) e_{\widetilde{A} \oplus \widetilde{B}}\left(t, t_{0}\right)\right. \\
\left.+\int_{t_{0}}^{t} e_{\widetilde{A} \oplus \widetilde{B}}(t, \sigma(s)) \widetilde{C}(s) \Delta s\right\}^{1 / p}
\end{gathered}
$$

for any $K>0, t \in I$

under the condition that $e_{\widetilde{A} \oplus \widetilde{B}}\left(T_{1}, t_{0}\right)<(\lambda+1) / \lambda$ and $\mu(t) B_{2}(t)<1$, where

$$
\begin{aligned}
\widetilde{A}(t) & =b(t)\left[\frac{q}{p} K^{(q-p) / p} v(\sigma(t), t)+\frac{r}{p}\right. \\
\cdot & K^{(r-p) / p} w(\sigma(t), t) \int_{t_{0}}^{t} g(\xi) \Delta \xi+\int_{t_{0}}^{t}\left(v_{t}^{\Delta}(t, s)\right. \\
+ & \left.\left.w_{t}^{\Delta}(t, s) \int_{t_{0}}^{s} g(\xi) \Delta \xi\right) \Delta s\right], \\
\widetilde{B}(t) & =\frac{B_{2}(t)}{1-\mu(t) B_{2}(t)}, \\
\widetilde{C}(t) & =[1+\mu(t) \widetilde{B}(t)] C_{2}(t), \\
B_{2}(t) & =b^{\Delta}(t) \int_{t_{0}}^{\sigma(t)}\left[\frac{q}{p} K^{(q-p) / p} v(\sigma(t), s)+\frac{r}{p}\right. \\
\cdot & \left.K^{(r-p) / p} w(\sigma(t), s) \int_{t_{0}}^{s} g(\xi) \Delta \xi\right] \Delta s, \\
C_{2}(t) & =b^{\Delta}(t) \int_{t_{0}}^{\sigma(t)}\left[\frac{p-q}{p} K^{q / p} v(\sigma(t), s)+\frac{p-r}{p}\right. \\
\cdot & \left.K^{r / p} w(\sigma(t), s) \int_{t_{0}}^{s} g(\xi) \Delta \xi\right] \Delta s+b(t)\left[\frac{p-q}{p}\right.
\end{aligned}
$$




$$
\begin{gathered}
\cdot K^{q / p} v(\sigma(t), t)+\frac{p-r}{p} K^{r / p} w(\sigma(t), t) \\
\cdot \int_{t_{0}}^{t} g(\xi) \Delta \xi+\int_{t_{0}}^{t}\left(\frac{p-q}{p} K^{q / p} v_{t}^{\Delta}(t, s)\right. \\
\left.\left.+\frac{p-r}{p} K^{r / p} w_{t}^{\Delta}(t, s) \int_{t_{0}}^{s} g(\xi) \Delta \xi\right) \Delta s\right], \\
\widetilde{D}\left(\lambda, T_{1}\right)=\frac{a\left(T_{1}\right)+\lambda \int_{t_{0}}^{T_{1}} e_{\widetilde{A} \oplus \widetilde{B}}\left(T_{1}, \sigma(s)\right) \widetilde{C}(s) \Delta s}{\lambda+1-\lambda e_{\widetilde{A} \oplus \widetilde{B}}\left(T_{1}, t_{0}\right)} .
\end{gathered}
$$

Proof. Define $z(t)$ by

$$
\begin{aligned}
& z(t)=a\left(T_{1}\right)+b(t) \int_{t_{0}}^{t}\left[v(t, s) u^{q}(\tau(s))\right. \\
& \left.+w(t, s) \int_{t_{0}}^{s} g(\xi) u^{r}(\xi) \Delta \xi\right] \Delta s+\lambda b\left(T_{1}\right) \\
& \quad \cdot \int_{t_{0}}^{T_{1}}\left[v\left(T_{1}, s\right) u^{q}(\tau(s))\right. \\
& \left.+w\left(T_{1}, s\right) \int_{t_{0}}^{s} g(\xi) u^{r}(\xi) \Delta \xi\right] \Delta s, \quad t \in I .
\end{aligned}
$$

Then, $z(t) \geq 0$ for $t \in I, z(t)$ is nondecreasing,

$$
u(t) \leq z^{1 / p}(t)
$$

and

$$
\begin{aligned}
& z\left(t_{0}\right)=a\left(T_{1}\right)+\lambda b\left(T_{1}\right) \int_{t_{0}}^{T_{1}}\left[v\left(T_{1}, s\right) u^{q}(\tau(s))\right. \\
& \left.+w\left(T_{1}, s\right) \int_{t_{0}}^{s} g(\xi) u^{r}(\xi) \Delta \xi\right] \Delta s .
\end{aligned}
$$

It is not difficult to obtain that

$$
u(\tau(t)) \leq z^{1 / p}(t)
$$

Taking the derivative of $z(t)$ and by Lemma 2, we get

$$
\begin{aligned}
& z^{\Delta}(t)=b^{\Delta}(t) \int_{t_{0}}^{\sigma(t)}\left[v(\sigma(t), s) u^{q}(s)\right. \\
& \left.+w(\sigma(t), s) \int_{t_{0}}^{s} g(\xi) u^{r}(\xi) \Delta \xi\right] \Delta s+b(t) \\
& \cdot\left[v(\sigma(t), t) u^{q}(\tau(t))+w(\sigma(t), t)\right. \\
& \left.\cdot \int_{t_{0}}^{t} g(\xi) u^{r}(\xi) \Delta \xi\right]+b(t) \\
& \cdot \int_{t_{0}}^{t}\left[v_{t}^{\Delta}(t, s) u^{q}(\tau(s))\right. \\
& \left.+w_{t}^{\Delta}(t, s) \int_{t_{0}}^{s} g(\xi) u^{r}(\xi) \Delta \xi\right] \Delta s .
\end{aligned}
$$

It follows from (33) and (35) that

$$
\begin{aligned}
& z^{\Delta}(t) \leq b^{\Delta}(t) \int_{t_{0}}^{\sigma(t)}\left[v(\sigma(t), s) z^{q / p}(s)\right. \\
& \left.+w(\sigma(t), s) \int_{t_{0}}^{s} g(\xi) z^{r / p}(\xi) \Delta \xi\right] \Delta s+b(t) \\
& \cdot\left[v(\sigma(t), t) z^{q / p}(t)+w(\sigma(t), t)\right. \\
& \left.\cdot \int_{t_{0}}^{t} g(\xi) z^{r / p}(\xi) \Delta \xi\right]+b(t) \\
& \cdot \int_{t_{0}}^{t}\left[v_{t}^{\Delta}(t, s) z^{q / p}(s)\right. \\
& \left.+w_{t}^{\Delta}(t, s) \int_{t_{0}}^{s} g(\xi) z^{r / p}(\xi) \Delta \xi\right] \Delta s .
\end{aligned}
$$

By Lemma 3, we get

$$
\begin{aligned}
& z^{q / p}(t) \leq \frac{q}{p} K^{(q-p) / p} z(t)+\frac{p-q}{p} K^{q / p}, \\
& z^{r / p}(t) \leq \frac{r}{p} K^{(r-p) / p} z(t)+\frac{p-r}{p} K^{r / p}
\end{aligned}
$$

for any $K>0$. Combining (37) with (38) yields

$$
\begin{aligned}
& z^{\Delta}(t) \leq z(\sigma(t)) b^{\Delta}(t) \int_{t_{0}}^{\sigma(t)}\left[\frac{q}{p} K^{(q-p) / p} v(\sigma(t), s)\right. \\
& \left.+\frac{r}{p} K^{(r-p) / p} w(\sigma(t), s) \int_{t_{0}}^{s} g(\xi) \Delta \xi\right] \Delta s+z(t) b(t) \\
& \cdot\left[\frac{q}{p} K^{(q-p) / p} v(\sigma(t), t)+\frac{r}{p} K^{(r-p) / p} w(\sigma(t), t)\right. \\
& \cdot \int_{t_{0}}^{t} g(\xi) \Delta \xi+\int_{t_{0}}^{t}\left(v_{t}^{\Delta}(t, s)\right. \\
& \left.\left.+w_{t}^{\Delta}(t, s) \int_{t_{0}}^{s} g(\xi) \Delta \xi\right) \Delta s\right]+b^{\Delta}(t) \\
& \int_{t_{0}}^{\sigma(t)}\left[\frac{p-q}{p} K^{q / p} v(\sigma(t), s)+\frac{p-r}{p}\right. \\
& \left.\cdot K^{r / p} w(\sigma(t), s) \int_{t_{0}}^{s} g(\xi) \Delta \xi\right] \Delta s+b(t)\left[\frac{p-q}{p}\right. \\
& \cdot K^{q / p} v(\sigma(t), t)+\frac{p-r}{p} K^{r / p} w(\sigma(t), t) \\
& \cdot \int_{t_{0}}^{t} g(\xi) \Delta \xi+\int_{t_{0}}^{t}\left(\frac{p-q}{p} K^{q / p} v_{t}^{\Delta}(t, s)\right. \\
& \left.\left.+\frac{p-r}{p} K^{r / p} w_{t}^{\Delta}(t, s) \int_{t_{0}}^{s} g(\xi) \Delta \xi\right) \Delta s\right]=\widetilde{A}(t) z(t) \\
& +B_{2}(t) z(\sigma(t))+C_{2}(t) .
\end{aligned}
$$


The remainder of the proof is similar to that of Theorem 4, and hence we omit it here.

Remark 10. In [14], we investigated the integral inequalities with mixed nonlinearities on time scales; however, the delay terms are not considered; furthermore, the inequalities considered in this paper are Volterra-Fredholm type. The results established in Theorems 4 and 9 generalize [14, Theorems 2.1 and 2.2].

\section{Application}

In this part, we present an example to illustrate the theoretical results.

Example 11. Consider the delay Volterra-Fredholm type integral equation on time scales

$$
\begin{aligned}
& u^{p}(t) \\
& =a(t) \\
& \quad+b(t) \int_{t_{0}}^{t} F\left(s, u(\tau(s)), \int_{t_{0}}^{s} G(\xi, u(\xi)) \Delta \xi\right) \Delta s \\
& \quad+\int_{t_{0}}^{T_{1}} H\left(s, u(\tau(s)), \int_{t_{0}}^{s} L(\xi, u(\xi)) \Delta \xi\right) \Delta s, \\
& \quad t \in I=\left[t_{0}, T_{1}\right]
\end{aligned}
$$

with the initial condition

$$
\begin{aligned}
u(t) & =\varphi(t), \quad \text { for } t \in\left[\alpha, t_{0}\right], \\
|\varphi(\tau(t))| & \leq|a(t)|^{1 / p}
\end{aligned}
$$

for every $t \in I$ with $\tau(t) \leq t_{0}$,

where $u(t)$ and $u(\tau(t))$ defined on the interval $I$ are the state and state delay variables, respectively. $a, b: I \longrightarrow \mathbb{R},|a|$ is nondecreasing, $b\left(T_{1}\right) \neq 0, T_{1} \in \mathbb{T}$, and $T_{1}>t_{0} . \tau: I \longrightarrow I$ satisfying $\tau(t) \leq t,-\infty<\alpha=\inf \{\tau(t), t \in I\} \leq t_{0}$, and $\varphi \in C_{r d}\left(\left[\alpha, t_{0}\right] \cap \mathbb{T}, \mathbb{R}\right), F, H: I \times \mathbb{R} \times \mathbb{R} \longrightarrow \mathbb{R}$, and $G, L:$ $I \times \mathbb{R} \longrightarrow \mathbb{R}$ are continuous functions.

Suppose that

$$
\begin{aligned}
|F(t, u, v)| & \leq f_{1}(t)|u|^{q}+f_{2}(t)|v|, \\
|G(t, u)| & \leq g(t)|u|^{r}, \\
|H(t, u, v)| & \leq f_{1}(t)|u|^{q}+f_{2}(t)|v|, \\
|L(t, u)| & \leq g(t)|u|^{r},
\end{aligned}
$$

where $p \geq q>0$ and $p \geq r>0$ are real constants and $f_{1}$, $f_{2}$, and $g$ are nonnegative rd-continuous functions on $I$. If
$e_{A \oplus B}\left(T_{1}, t_{0}\right)<1+\left|b\left(T_{1}\right)\right|$ and $\mu(t) B_{1}(t)<1$, then the solution $u(t)$ of (40) satisfies

$$
\begin{gathered}
u(t) \leq\left\{D\left(\lambda, T_{1}\right) e_{A \oplus B}\left(t, t_{0}\right)\right. \\
\left.+\int_{t_{0}}^{t} e_{A \oplus B}(t, \sigma(s)) C(s) \Delta s\right\}^{1 / p}
\end{gathered}
$$

for any $K>0, t \in I$,

where

$$
\begin{aligned}
& A(t)=b(t)\left[\frac{q}{p} K^{(q-p) / p} f_{1}(t)+\frac{r}{p} K^{(r-p) / p} f_{2}(t)\right. \\
& \left.\cdot \int_{t_{0}}^{t} g(\xi) \Delta \xi\right] \\
& B(t)=\frac{B_{1}(t)}{1-\mu(t) B_{1}(t)}, \\
& C(t)=[1+\mu(t) B(t)] C_{1}(t), \\
& B_{1}(t)=b^{\Delta}(t) \int_{t_{0}}^{\sigma(t)}\left[\frac{q}{p} K^{(q-p) / p} f_{1}(s)\right. \\
& \left.+\frac{r}{p} K^{(r-p) / p} f_{2}(s) \int_{t_{0}}^{s} g(\xi) \Delta \xi\right] \Delta s, \\
& C_{1}(t)=b^{\Delta}(t) \int_{t_{0}}^{\sigma(t)}\left[\frac{p-q}{p} K^{q / p} f_{1}(s)\right. \\
& \left.+\frac{p-r}{p} K^{r / p} f_{2}(s) \int_{t_{0}}^{s} g(\xi) \Delta \xi\right] \Delta s+b(t)\left[\frac{p-q}{p}\right. \\
& \left.\cdot K^{q / p} f_{1}(t)+\frac{p-r}{p} K^{r / p} f_{2}(t) \int_{t_{0}}^{t} g(\xi) \Delta \xi\right] \text {, } \\
& D\left(\lambda, T_{1}\right) \\
& =\frac{\left|a\left(T_{1}\right) b\left(T_{1}\right)\right|+\int_{t_{0}}^{T_{1}} e_{A \oplus B}\left(T_{1}, \sigma(s)\right) C(s) \Delta s}{1+\left|b\left(T_{1}\right)\right|-e_{A \oplus B}\left(T_{1}, t_{0}\right)} .
\end{aligned}
$$

Actually, by (40), $u(t)$ satisfies

$$
\begin{aligned}
& |u(t)|^{p} \leq|a(t)|+|b(t)| \\
& \cdot \int_{t_{0}}^{t}\left|F\left(s, u(\tau(s)), \int_{t_{0}}^{s} G(\xi, u(\xi)) \Delta \xi\right)\right| \Delta s \\
& \quad+\int_{t_{0}}^{T_{1}}\left|H\left(s, u(\tau(s)), \int_{t_{0}}^{s} L(\xi, u(\xi)) \Delta \xi\right)\right| \Delta s \\
& \quad \leq|a(t)|+|b(t)| \int_{t_{0}}^{t}\left[f_{1}(s)|u(\tau(s))|^{q}\right. \\
& \left.\quad+f_{2}(s) \int_{t_{0}}^{s} g(\xi)|u(\xi)|^{r} \Delta \xi\right] \Delta s
\end{aligned}
$$




$$
\begin{aligned}
& +\int_{t_{0}}^{T_{1}}\left[f_{1}(s)|u(\tau(s))|^{q}\right. \\
& \left.+f_{2}(s) \int_{t_{0}}^{s} g(\xi)|u(\xi)|^{r} \Delta \xi\right] \Delta s, \quad t \in I .
\end{aligned}
$$

It is not difficult to verify (43) is satisfied by Corollary 8 .

\section{Data Availability}

No data were used to support this study.

\section{Conflicts of Interest}

The authors declare that they have no conflicts of interest.

\section{Authors' Contributions}

All three authors contributed equally to this work. They all read and approved the final version of the manuscript.

\section{Acknowledgments}

This work was supported by the National Natural Science Foundations of China (11671227, 61703180), the Natural Science Foundation of Shandong Province (ZR2017LF012), A Project of Shandong Province Higher Educational Science and Technology Program (J17KA157), and the Doctoral Scientific Research Foundation of University of Jinan (1008398).

\section{References}

[1] S. Hilger, "Analysis on measure chains: a unified approach to continuous and discrete calculus," Results in Mathematics, vol. 18, no. 1-2, pp. 18-56, 1990.

[2] M. Bohner and A. Peterson, Dynamic Equations on Time Scales: An Introduction with Applications, Birkhauser, Boston, MA, USA, 2001.

[3] Y. Tian, Y. Cai, and Y. Sun, "Asymptotic behavior of switched delay systems with nonlinear disturbances," Applied Mathematics and Computation, vol. 268, pp. 522-533, 2015.

[4] B. Ben Nasser, K. Boukerrioua, M. Defoort, M. Djemai, and M. A. Hammami, "State feedback stabilization of a class of uncertain nonlinear systems on non-uniform time domains," Systems \& Control Letters, vol. 97, pp. 18-26, 2016.

[5] R. P. Agarwal, D. O'Regan, and S. Saker, Dynamic Inequalities on Time Scales, Springer International Publishing, Basel, Switzerland, 2014.

[6] F. C. Jiang and F. W. Meng, "Explicit bounds on some new nonlinear integral inequalities with delay," Journal of Computational and Applied Mathematics, vol. 205, no. 1, pp. 479-486, 2007.

[7] Q. Feng, F. Meng, and B. Zheng, "Gronwall-Bellman type nonlinear delay integral inequalities on time scales," Journal of Mathematical Analysis and Applications, vol. 382, no. 2, pp. 772784, 2011.

[8] J. Gu and F. Meng, "Some new nonlinear Volterra-Fredholm type dynamic integral inequalities on time scales," Applied Mathematics and Computation, vol. 245, pp. 235-242, 2014.
[9] H. Liu, "A class of retarded Volterra-Fredholm type integral inequalities on time scales and their applications," Journal of Inequalities and Applications, Paper No. 293, 15 pages, 2017.

[10] H. Liu, "Some new integral inequalities with mixed nonlinearities for discontinuous functions," Advances in Difference Equations, Paper No. 22, 16 pages, 2018.

[11] W. N. Li, "Some new dynamic inequalities on time scales," Journal of Mathematical Analysis and Applications, vol. 319, no. 2, pp. 802-814, 2006.

[12] Q. H. Ma and J. Pečarić, "The bounds on the solutions of certain two-dimensional delay dynamic systems on time scales," Computers \& Mathematics with Applications. An International Journal, vol. 61, no. 8, pp. 2158-2163, 2011.

[13] Y. Tian, M. Fan, and F. Meng, "A generalization of retarded integral inequalities in two independent variables and their applications," Applied Mathematics and Computation, vol. 221, pp. 239-248, 2013.

[14] Y. Tian, Y. Cai, L. Li, and T. Li, "Some dynamic integral inequalities with mixed nonlinearities on time scales," Journal of Inequalities and Applications, vol. 2015, article 12, pp. 1-10, 2015.

[15] Y. Tian, M. Fan, and Y. Sun, "Certain nonlinear integral inequalities and their applications," Discrete Dynamics in Nature and Society, vol. 2017, Article ID 8290906, 8 pages, 2017.

[16] R. P. Agarwal, S. Deng, and W. Zhang, "Generalization of a retarded Gronwall-like inequality and its applications," Applied Mathematics and Computation, vol. 165, no. 3, pp. 599-612, 2005.

[17] A. Abdeldaim and A. A. El-Deeb, "On generalized of certain retarded nonlinear integral inequalities and its applications in retarded integro-differential equations," Applied Mathematics and Computation, vol. 256, pp. 375-380, 2015.

[18] A. A. El-Deeb and R. G. Ahmed, "On some generalizations of certain nonlinear retarded integral inequalities for VolterraFredholm integral equations and their applications in delay differential equations," Journal of the Egyptian Mathematical Society, vol. 25, no. 3, pp. 279-285, 2017.

[19] R. Xu and F. Meng, "Some new weakly singular integral inequalities and their applications to fractional differential equations," Journal of Inequalities and Applications, vol. 2016, no. 78, 2016.

[20] R. Xu and X. Ma, "Some new retarded nonlinear VolterraFredholm type integral inequalities with maxima in two variables and their applications," Journal of Inequalities and Applications, vol. 2017, article no. 187, 2017.

[21] W.-S. Wang, X. Zhou, and Z. Guo, "Some new retarded nonlinear integral inequalities and their applications in differential-integral equations," Applied Mathematics and Computation, vol. 218, no. 21, pp. 10726-10736, 2012.

[22] X.-L. Ding and B. Ahmad, "A generalized Volterra-Fredholm integral inequality and its applications to fractional differential equations," Advances in Difference Equations, Paper No. 91, 8 pages, 2018.

[23] A. Boudeliou, "On certain new nonlinear retarded integral inequalities in two independent variables and applications," Applied Mathematics and Computation, vol. 335, pp. 103-111, 2018.

[24] S. H. Saker, "Applications of Opial inequalities on time scales on dynamic equations with damping terms," Mathematical and Computer Modelling, vol. 58, no. 11-12, pp. 1777-1790, 2013.

[25] O. Abu Arqub, "An iterative method for solving fourth-order boundary value problems of mixed type integro-differential equations," Journal of Computational Analysis and Applications, vol. 18, no. 5, pp. 857-874, 2015. 
[26] M. Al-Smadi, O. A. Arqub, N. Shawagfeh, and S. Momani, "Numerical investigations for systems of second-order periodic boundary value problems using reproducing kernel method," Applied Mathematics and Computation, vol. 291, pp. 137-148, 2016.

[27] O. Abu Arqub, "Fitted reproducing kernel Hilbert space method for the solutions of some certain classes of time-fractional partial differential equations subject to initial and Neumann boundary conditions," Computers \& Mathematics with Applications, vol. 73, no. 6, pp. 1243-1261, 2017.

[28] F. Ghomanjani, M. H. Farahi, and N. Pariz, "A new approach for numerical solution of a linear system with distributed delays, VOLterra delay-integro-differential equations, and nonlinear VOLterra-Fredholm integral equation by Bezier curves," Computational \& Applied Mathematics, vol. 36, no. 3, pp. 1349-1365, 2017. 


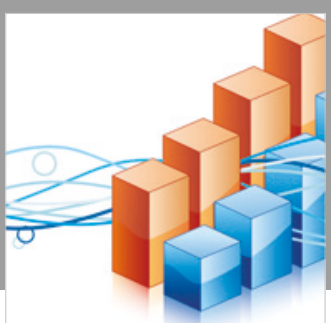

Advances in

Operations Research

\section{-n-m}
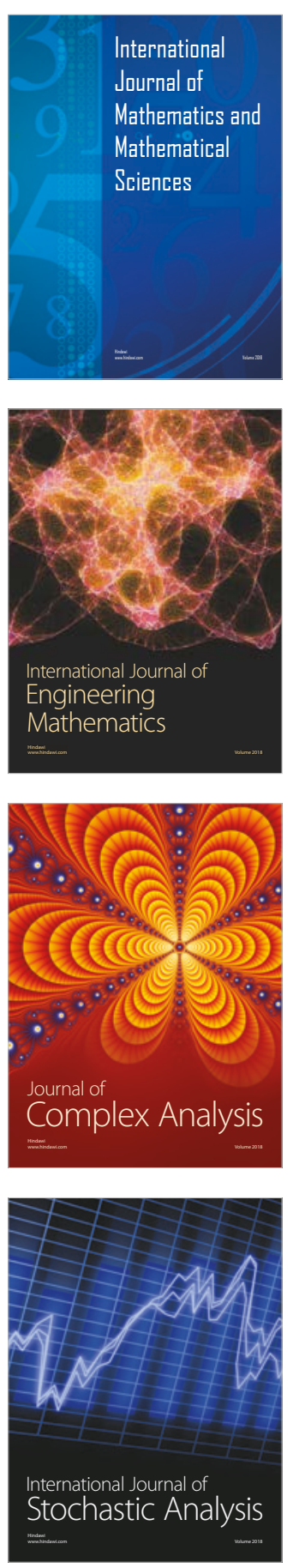
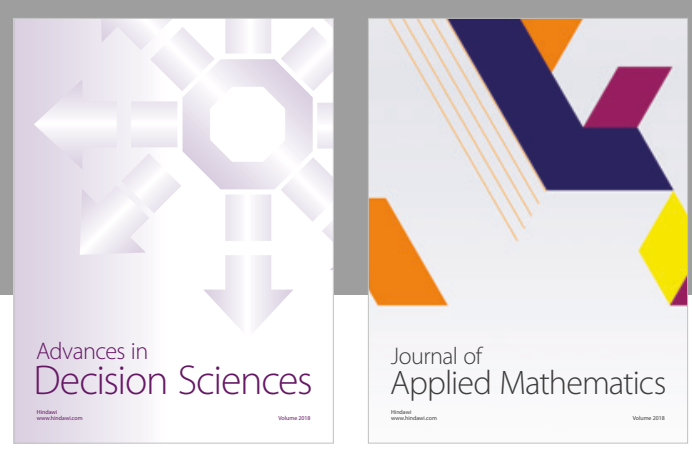

Journal of

Applied Mathematics
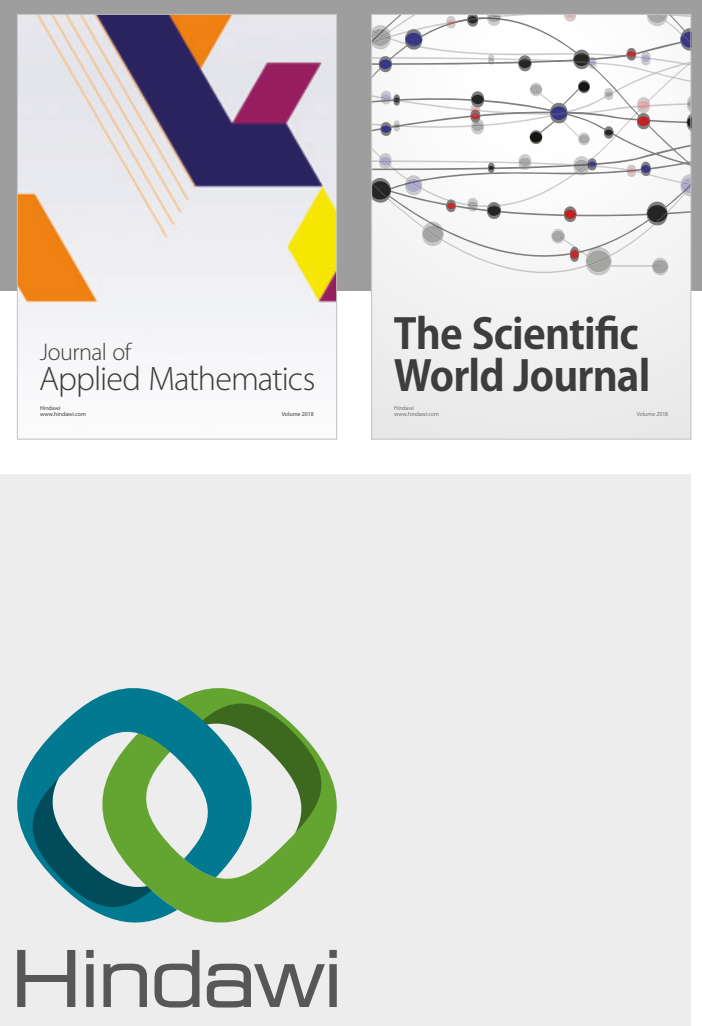

Submit your manuscripts at

www.hindawi.com

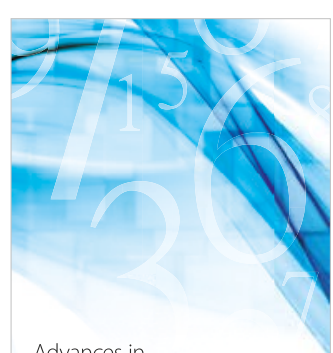

Advances in
Numerical Analysis
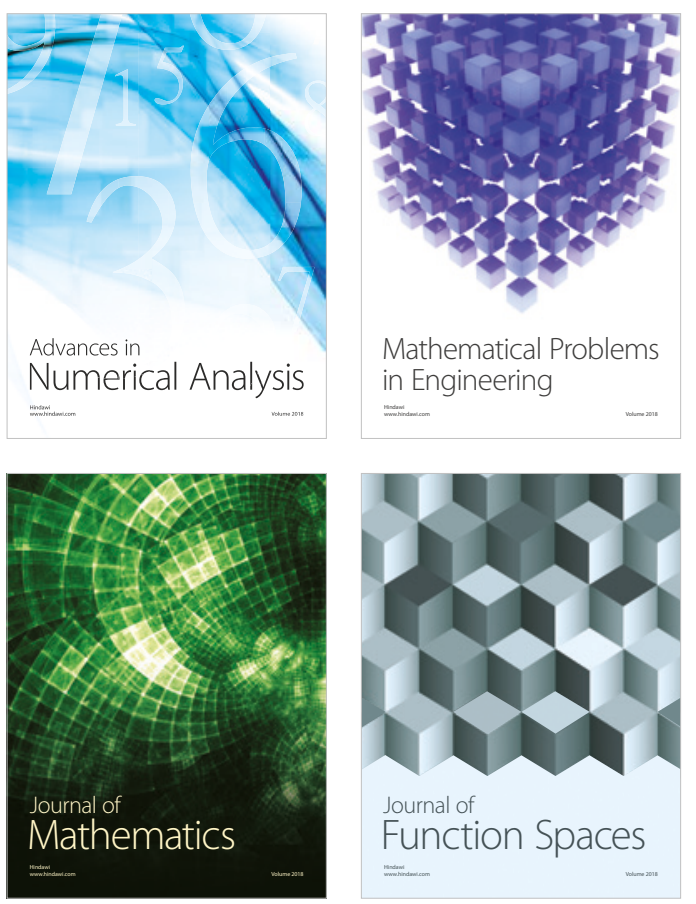

Mathematical Problems in Engineering

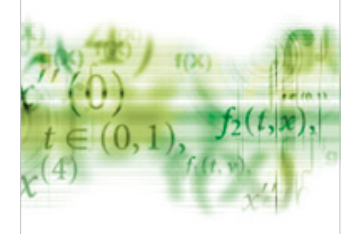

International Journal of

Differential Equations

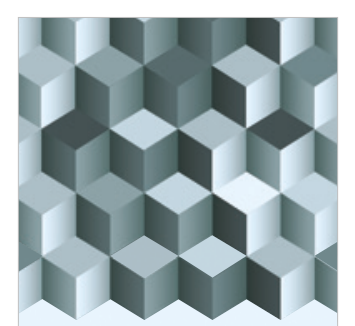

Journal of

Function Spaces

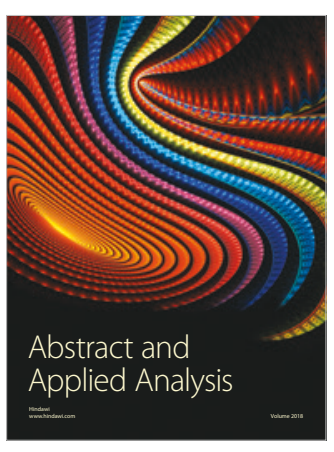

The Scientific

World Journal

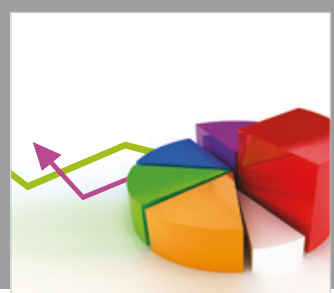

Journal of

Probability and Statistics
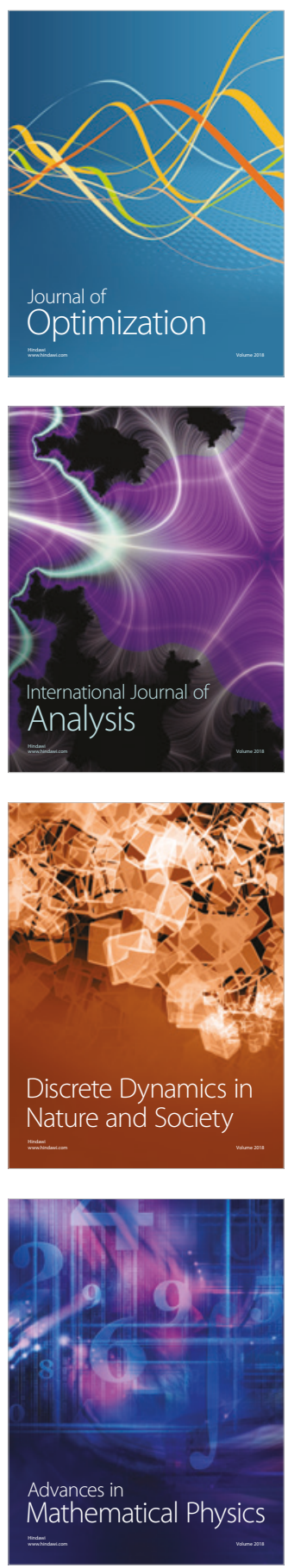